\title{
Orocutaneous fistulas of odontogenic origin presenting as a recurrent pyogenic granuloma
}

\author{
Jin Hoon Lee, \\ Jae Wook Oh, \\ Sung Ho Yoon \\ Department of Plastic and \\ Reconstructive Surgery, Inje University \\ Haeundae Paik Hospital, Busan, Korea
}

\begin{abstract}
Orocutaneous fistulas, or cutaneous sinuses of odontogenic origin, are uncommon but often misdiagnosed as skin lesions unrelated to dental origin by physicians. Accurate diagnosis and use of correct investigative modalities are important because orocutaneous fistulas are easily confused for skin or bone tumors, osteomyelitis, infected cysts, salivary gland fistulas, and other pathologies. The aim of this study is to present our experience with a patient with orocutaneous fistulas of odontogenic origin presenting as recurrent pyogenic granuloma of the cheek, and to discuss their successful treatment.
\end{abstract}

Keywords: Cutaneous fistula / Pyogenic granuloma / Recurrence

\section{INTRODUCTION}

Orocutaneous fistulas, or cutaneous sinuses of odontogenic origin, are a rare condition and diagnosis is challenging. They are often misdiagnosed by physicians as skin lesions or nonodontogenic lesion because they are easily confused for skin or bone tumors, osteomyelitis, infected cysts, salivary gland fistulas, and other pathologies [1]. We present a patient with orocutaneous fistulas presenting as a recurrent pyogenic granuloma (PG) of the cheek, which was initially misdiagnosed as a nonodontogenic lesion. We emphasize that accurate diagnosis and use of correct investigative modalities are important for prompt treatment and for reducing recurrence and complication rates.

\section{CASE REPORT}

A 74-year-old female patient visited Inje University Haeundae Paik Hospital with a chief complaint of a pinkish mass on her right cheek of 3 months' duration. A history of the presenting

\footnotetext{
Correspondence: Sung Ho Yoon

Department of Plastic and Reconstructive Surgery, Inje University Haeundae Paik Hospital, Inje University College of Medicine, 875 Haeun-daero, Haeundae-gu, Busan 48108, Korea

E-mail: y34805@korea.com

Received June 29, 2018 / Revised September 30, 2018 / Accepted October 11, 2018
}

illness revealed that the growth was gradual in onset, and mass was initially small but grew to the present size. The growth measured approximately $0.3 \mathrm{~cm} \times 0.3 \mathrm{~cm}$ in diameter and was soft to firm in consistency (Fig. 1). The skin lesion was painless and asymptomatic, except for the slight discomfort of the patient due to the growth. There was no evidence of pus discharge from the lesion. Intraoral examination showed no other mass lesions, fistula, or gingivitis. No relevant medical history was obtained from the patient and her family.

The skin lesion was completely excised under local anesthesia and sent for histopathological examination. The subsequent skin defect was covered by bilateral V-Y advancement flaps. Fig. 2 shows the immediate postoperative appearance, which confirms that the skin defect was covered without any tension. Histopathologically, hematoxylin and eosin staining confirmed the diagnosis of PG (Fig. 3). The healing period was uneventful, but 2 months after surgery, the patient returned to our clinic with persistent discharge from the previous wound site. A second surgery was performed to excise the recurrent skin lesion. A unilateral V-Y advancement flap was used for the coverage of the skin defect (Fig. 4). Similar to the initial surgery, there was no problem with wound healing. However, 1 month later, she returned to our clinic with a complaint of persistent discharge. A wound culture test was performed but no bacteria were iden- 


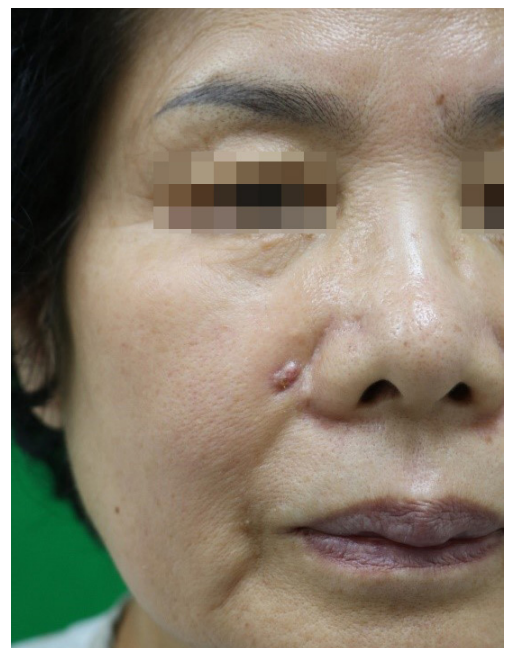

Fig. 1. A 74-year-old woman with a pinkish soft mass in her right cheek region.

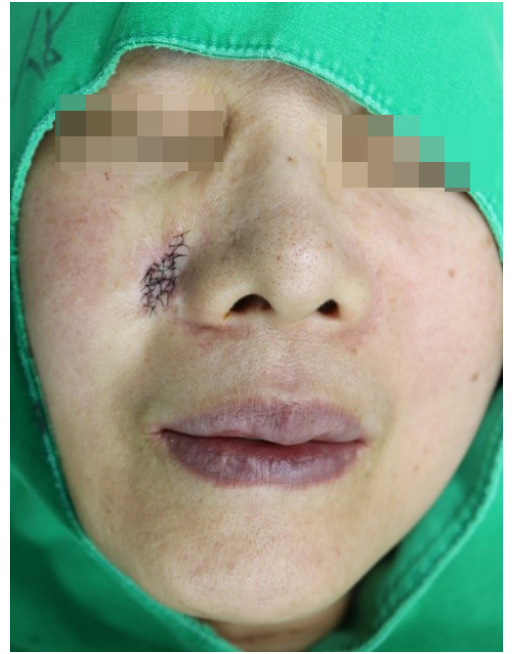

Fig. 2. Immediate postoperative photo showing complete elimination of the mass by the bilateral V-Y advancement flap.

tified. For histopathological examination, a tissue biopsy was performed and the results revealed chronic inflammation and abscess formation.

In order to identify the cause of the recurrent PG, intraoral examination was repeated. There were no findings suggestive of odontogenic infection. Subsequently, panoramic X-ray and computed tomography (CT) were performed. The panoramic radiograph revealed partial alveolar bone loss due to pulpitis and the presence of a periapical abscess of the right maxillary canine tooth (Fig. 5). However, there were no findings suggestive of chronic osteomyelitis, such as soft tissue swelling, mucosal abscess, cutaneous abscess, and draining fistulas, on the CT images (Fig. 6). For evaluation of the odontogenic infection, the patient was referred to the dental department. During dental

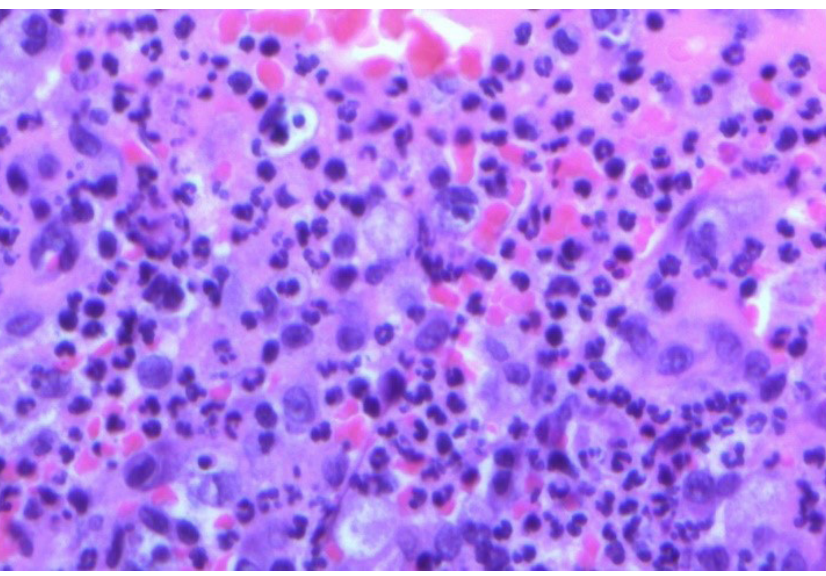

Fig. 3. Histopathologic examination of the lesion shows that it consists of condensed small-caliber vessels with scattered red blood cells. The stroma is fibromyxoid with scattered inflammatory cells. Cytologic atypia and mitoses are observed $(\mathrm{H} \& \mathrm{E}, \times 400)$.

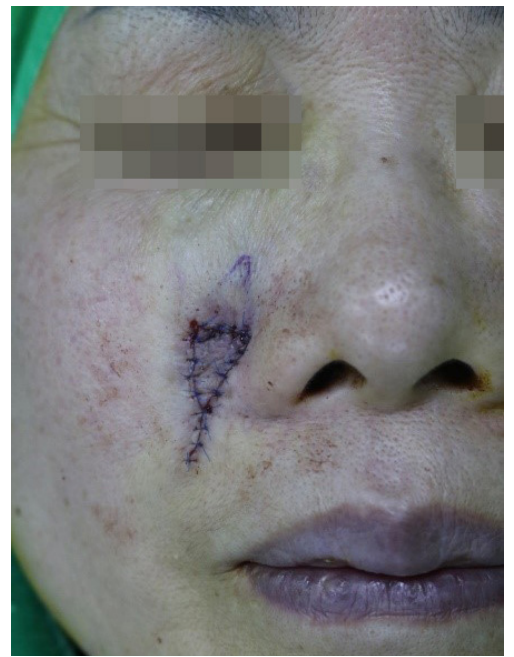

Fig. 4. Secondary postoperative photo showing coverage with a unilateral V-Y advancement flap.

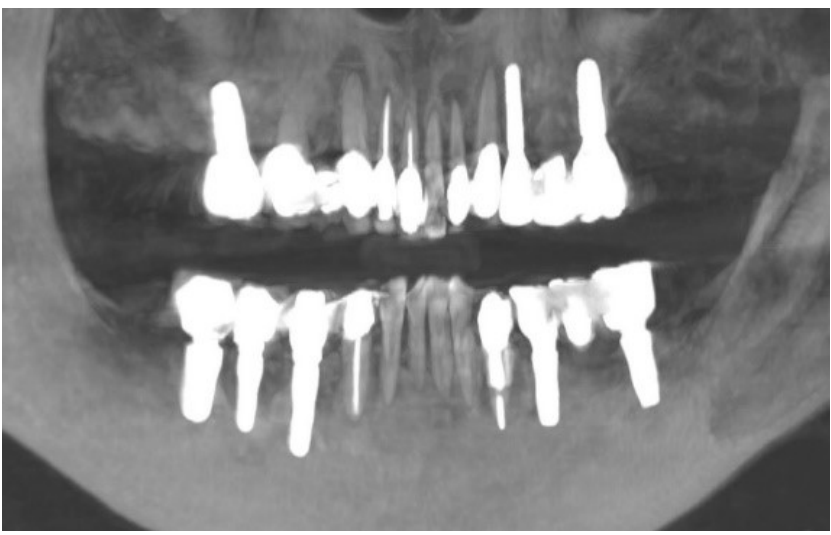

Fig. 5. Panoramic view of dental computed tomography images showing partial alveolar bone loss and periapical abscess formation in the right maxillary canine teeth. 

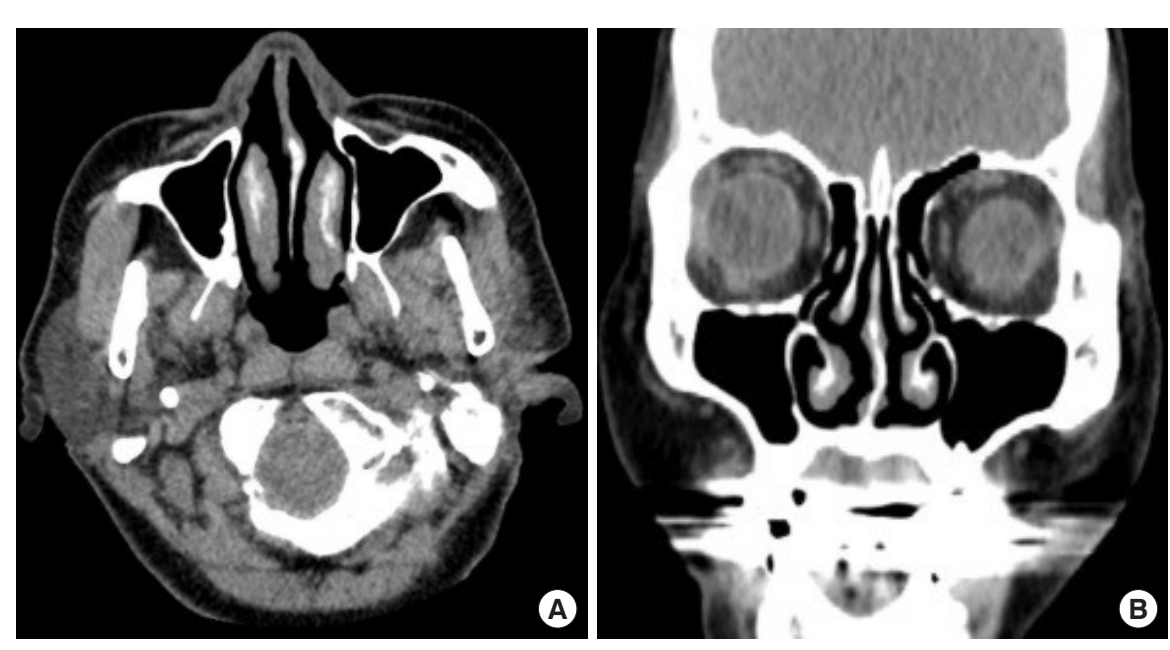

Fig. 6. Facial computed tomography images of the cheek region. (A) Axial view. (B) Coronal view.

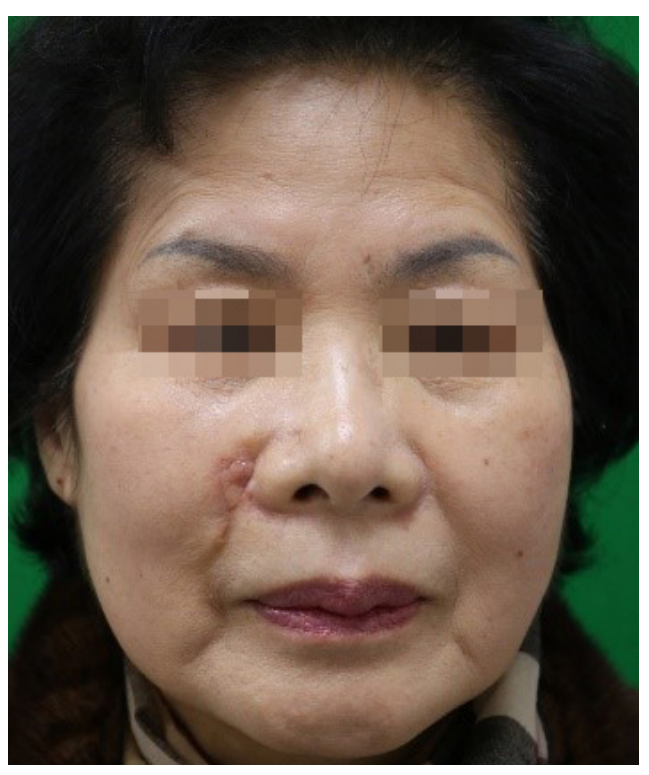

Fig. 7. Postoperative photo 3 months after extraction of the right maxillary canine tooth.

evaluation, irreversible pulpitis was observed on the right maxillary canine tooth. Therefore, the tooth was extracted and systemic antimicrobial therapy was started to suppress the odontogenic infection. The patient visited our clinic 2 weeks after the extraction, and no secretions from the cheek flap site were found and wound healing was complete. After 3 months, all odontogenic infections had resolved and no further recurrence was observed (Fig. 7). The extracted tooth was replaced with a dental implant.

\section{DISCUSSION}

PG, also known as lobular capillary hemangioma, is a benign proliferative vascular tumor of the skin or mucous membranes that is characterized by rapid growth and a friable surface [2].
In fact, the term "pyogenic granuloma" is a misnomer as it does not contain pus and is not, strictly speaking, a granuloma histopathologically [3]. Although the cause of PG is unknown, it is thought to be a hyperplastic and neovascular response to angiogenic stimuli, such as chronic low-grade local irritation, traumatic injury, hormonal factors, or certain drugs [4].

Clinically, PG is a smooth or lobulated exophytic lesion manifesting as a small, red, erythematous papule on a pedunculated or sometimes sessile base, which is usually hemorrhagic and compressible [5-7]. The size varies in diameter from a few millimeters to several centimeters, but is usually less than $2.5 \mathrm{~cm}$ [8]. It can grow rapidly and will often bleed profusely with little or no trauma. It may be found in the oral cavity or extraorally. Intraorally, the gingiva is the most common site of involvement (approximately 60\%-70\%), followed by the lips (14\%), tongue (9\%), buccal mucosa (7\%), and palate (2\%) [9]. Histopathologic confirmation is helpful for making a definitive diagnosis. There are several different treatment modalities, such as surgical excision, which is the treatment of choice, and laser therapy, electrocautery, cryotherapy, sclerotherapy, steroid injection, and intralesional therapies using chemicals [4]. Recurrence is observed in up to $16 \%$ of patients after surgical excision of the PG lesions and secondary operations are mandatory [5-7]. This is believed to result from incomplete excision, failure to eliminate etiologic factors, or reinjury to the area. Therefore, the stimulating factors, if present, should be prevented, eliminated, or discontinued prior to the treatment to ensure successful outcomes. Our patient had a recurrent PG of the cheek that recurred even after secondary excision. The patient had dental complications that caused loss of the alveolar bone and periapical abscess formation. The development of PG may have resulted from the persistent local trauma in the gap between the alveolar bone and canine tooth. In addition, the lack of adequate keratinized mucosa may have allowed the entrapment of soft tissue inside 
the gap, and contributed to the development of the PG. PGs associated with dental complications are thought to be capable of disseminating to the cheek region, causing external skin lesions. After the canine tooth was extracted and the odontogenic infection was completely resolved, the cheek flap healed spontaneously without recurrence.

Orocutaneous fistulas of odontogenic origin are often misdiagnosed by physicians and confused for skin or bone tumors, osteomyelitis, infected cysts, salivary gland fistulas, and other pathologies [1]. Our successful treatment strategy emphasizes the importance of the diagnosis with careful history-taking, meticulous examination, and radiographic examination with the awareness of odontogenic origin. In conclusion, treatment of orocutaneous fistulas of odontogenic origin should be combined with the elimination of any present provoking factors to ensure a successful outcome and prevent recurrence.

\section{NOTES}

\section{Conflict of interest}

No potential conflict of interest relevant to this article was reported.

\section{Ethical approval}

The study was performed in accordance with the principles of the Declaration of Helsinki. Written informed consent was obtained.

\section{Patient consent}

The patient provided written informed consent for the publication and the use of her images.

\section{ORCID}

Jin Hoon Lee https://orcid.org/0000-0003-3938-3427
Jae Wook Oh

https://orcid.org/0000-0001-8829-0935

Sung Ho Yoon

\section{REFERENCES}

1. Sadeghi S, Dibaei M. Prevalence of odontogenic sinus tracts in 728 endodontically treated teeth. Med Oral Patol Oral Cir Bucal 2011;16:e296-9.

2. Mills SE, Cooper PH, Fechner RE. Lobular capillary hemangioma: the underlying lesion of pyogenic granuloma: a study of 73 cases from the oral and nasal mucous membranes. Am J Surg Pathol 1980;4:470-9.

3. Patil K, Mahima VG, Lahari K. Extragingival pyogenic granuloma. Indian J Dent Res 2006;17:199-202.

4. Jafarzadeh H, Sanatkhani M, Mohtasham N. Oral pyogenic granuloma: a review. J Oral Sci 2006;48:167-75.

5. Eversole LR. Clinical outline of oral pathology: diagnosis and treatment. 3rd ed. Hamilton: BC Decker; 2002.

6. Neville BW, Damm DD, Allen CM, Bouquot JE. Oral and maxillofacial pathology. 2nd ed. Philadelphia: W.B. Saunders; 2002.

7. Regezi JA, Sciubba JJ, Jordan RC. Oral pathology: clinical pathologic considerations. 4th ed. Philadelphia: W.B. Saunders; 2003.

8. Gordon-Nunez MA, de Vasconcelos Carvalho M, Benevenuto TG, Lopes MF, Silva LM, Galvao HC. Oral pyogenic granuloma: a retrospective analysis of 293 cases in a Brazilian population. J Oral Maxillofac Surg 2010;68:2185-8.

9. Al-Shamiri HM, Alaizari NA, Al-Maweri SA, Tarakji B. Development of pyogenic granuloma and hemangioma after placement of dental implants: a review of literature. J Int Soc Prev Community Dent 2015;5:77-80. 